\title{
Optical Wireless Access Point Agent Networks
}

\author{
Tae-Gyu Lee* \\ Division of Computer Engineering, School of Electrical \& Computer Engineering, Chungbuk \\ National University, Cheongju, Chungbuk 361-763, Korea
}

(Received February 2, 2009 : revised February 26, 2009 : accepted February 27, 2009)

\begin{abstract}
This paper proposes an optical wireless transfer agent method which realizes the continuous and swift data transfer of optical wireless terminals in optical wireless networks. The unguided wireless channel generally shows frequent link disconnections and propagation delays due to weak wireless links. Specially speaking, optical wireless channels have more vulnerable links and roaming propagation delays relative to the weakness of the previous RF channels due to their low signal connectivity and small geographic coverage. Conventional optical wireless network protocols did not consider any fault models about physical link faults. Consequently, they have shown data transfer inefficiency for both data link control and physical wireless link control. To overcome these optical wireless environmental problems, this paper suggests a new wireless access point (or base station) agent system, which provides wireless or mobile clients with previous link layer protocols compensated.
\end{abstract}

Keywords : Optical wireless, Transfer protocols, Fault tolerant, Disconnected operation, Agent systems

OCIS codes : (060.0060) Fiber optics and optical communications; (060.4250) Networks; (060.4510) Optical communications; (000.3870) Mathematics; (040.3060) Infrared

\section{INTRODUCTION}

Today's advanced wireless network environment accelerates user desire for contents and service quality. Wireless handheld terminals have been extending their service coverage into bidirectional video interactive services. These service trends boost the available resource requirements of hardware or software such as broadband wireless channel bandwidth and large battery and memory capacity. But the constraint requirements are not yet met even though technology is continuously upgraded by technical advances.

Moreover optical wireless networks combined with wired Internet is attractive as a future ultra or superultra speed data transfer environment (such as Gbps rates or higher) considering wireless local area networks as well as wireless global networks $[1,2]$. So far, optical wireless networks are primarily applied to the configuration of wireless local transfer systems based on Infrared or IrDA specification. Independent of issues of physical medium, optical wireless networks reveal themselves in

*Corresponding author: tigerlee88@empal.com the fact that we make good use of ultrahigh speed channels and security advantages such as Giga-IR [3-6].

This paper provides a platform structure and advance mechanisms for optical wireless communications to support real-time data transfer services by instant commands to mobile users. Optical wireless channels are frequently disconnected or delayed in time due to environmental factors such as weak wireless channels and terminal resources. Together, by overcoming the restrictions, this paper proposes an innovative agent system, advanced transfer protocol and packet loss preservation method for real-time data transfer.

In this paper, I will consider the state of channel disconnection as link failure on a physical layer. A relevant link fault model is defined and disconnection problem issues are described. Additionally, a link fault detection method is devised and a wireless access point (or base station) agent method is proposed as a component of a fault tolerant system.

This paper only applies Infrared or IrDA specification as the physical medium into an optical wireless LAN model, and then it shall be expanded into optical-wireless WAN as a global network model for expandability or 
compatibility by continuous studies $[7,8]$.

This paper is organized as follows. Section II describes related works of an optical wireless network system. Section III demonstrates a reference system model and connectivity definitions for an optical wireless network system. Section IV presents an optical wireless access point transfer agent to transmit data with disconnected operations on optical wireless networks. Next, Section $\mathrm{V}$ analyzes and evaluates system characteristics and performances of a proposed agent system. Finally, Section VI sums up with conclusions and future directions.

\section{RELATED WORKS}

Present optical wireless networks mainly provide indoor communications as wireless local networks. Future ones have new related issues of multi-channel communication, broadband communication and global network configuration $[1,4,5,9]$.

Then data packet relays and linkages are researched for wireless to wired communications. A wireless sending terminal transforms its wireless packets into IP based packets of the third layer through wireless data gateways to transfer to wired servers or wireless receiving terminals on the remote Internet [6].

Previous transfer protocols and mechanisms have raised issues of inefficiency and problems because of long reconnection times and retry transmissions on TCP layers applied to physical link disconnection. These issues have brought about time delays of packet propagation, data packet losses and transfer channel inefficiency, etc. [11].

Moreover, a data link layer uses retry and retransmission mechanisms based on ARQ-like methods with timeouts (or time bounds) to overcome the network disconnection or packet loss $[12,13]$.

Also, a physical layer protocol has proposed a network architecture alternative for resetting or rerouting network links to tolerate physical link failures $[10,14]$. These previous works did not have error detect or error control mechanisms provided for managing physical link failures on the data link layer as well as physical layers or any other layers.

Optical wireless data transfer supports IrBurst specification which would transmit large volumes of multimedia data including multimedia-text, audio, video and other multimedia with high real-time $[3,4,15]$. However, these past research projects cannot provide mobile clients with efficient QoS due to frequent disconnection and reconnection of wireless terminals in optical wireless networks.

This work newly describes the definition of frequent transient or permanent disconnections by physical or logical link of optical wireless networks, and presents an advanced alternative for improving packet loss, transfer delay and channel efficiency. One could not see modeling, detecting or coping with physical link disconnection on a physical layer.

This paper proposes an agent-based transfer method which guarantees QoS of real-time data transfer despite disconnecting and reconnecting during roaming of optical wireless terminals. The proposed method is an agent technique which improves packet receiving ratio, data transfer efficiency and transfer delay more over the 2nd layer (or data link layer) of OSI or TCP/IP protocols.

\section{OPTICAL WIRELESS NETWORK SYSTEM}

This work constructs a system model for real-time transfer services and proposes as safer and more efficient real-time data transfer method based on a client/server model in optical wireless network. We use a unified model which does not differentiate link disconnection from a mobile client roaming considering the link fault model. But we can divide the unified model into two separate models, which contain semantically more details from a mobility point of view.

The mobile client downloads the various real-time data from the data transfer server in optical wireless data transfer as Fig. 1.

The 802.11 MAC layer is responsible for how a client associates with an access point (AP). When an 802.11 client enters the range of one or more APs, it chooses an access point to associate with (also called joining a Basic Service Set). This access point is based on signal strength and observed packet error rates. Once accepted by the access point, the client tunes to the radio channel to which the access point is set. Periodically it surveys all 802.11 channels in order to assess whether a different access point would provide it with better performance characteristics. If this turns out to be the case, it reassociates with the new access point, tuning to the radio channel to which that access point is set (Fig. 1).

Reassociation usually occurs because the wireless station has physically moved away from the original access

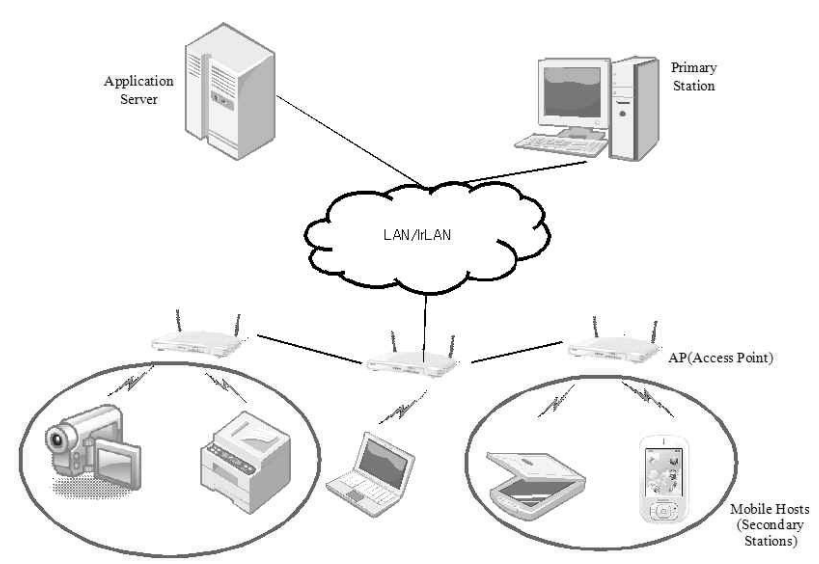

FIG. 1. Optical wireless network hierarchy. 

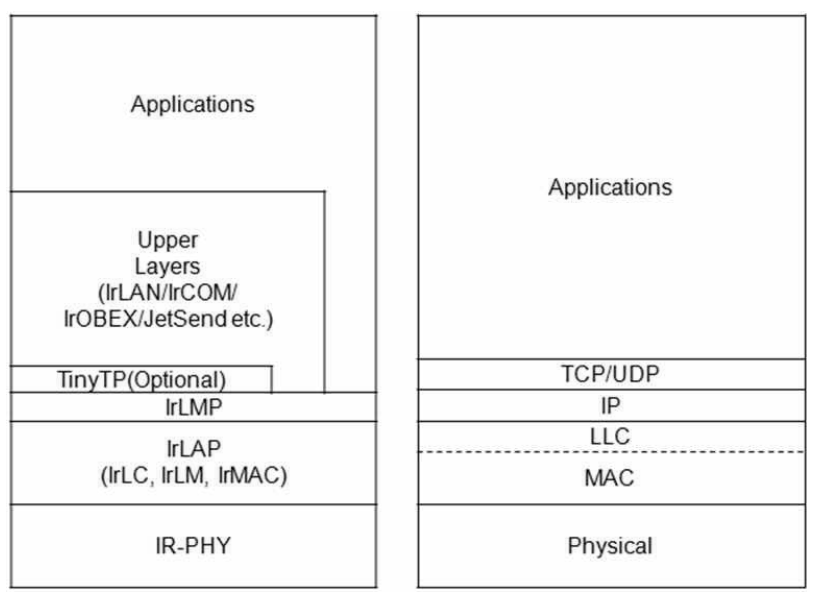

FIG. 2. IrDA versus TCP/IP protocol stacks.
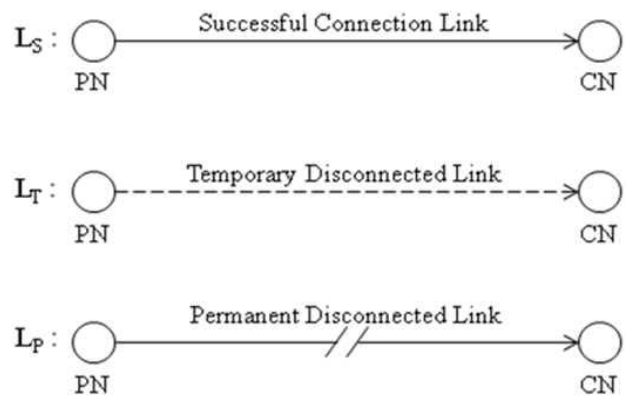

OPN: Provider Node or Prim ary Station OCN: Client Node or Secondary Station

FIG. 3. Link connectivity models.

point, causing the signal to weaken. In other cases, reassociation occurs due to a change in radio characteristics in the building, or due simply to high network traffic on the original access point. In the latter case this function is known as "load balancing," since its primary function is to distribute the total WLAN load most efficiently across the available wireless infrastructure.

As Fig. 2 this research is the agent method that improves flow and an error control protocol in which is a data link layer IrLAP or an application network layer IrLAN in IrDA/IrSimple transmission protocol stacks. Further it raises the transmission efficiency of TCP and UDP protocols which are a transport layer over the existing TCP/IP protocol stack.

Optical wireless packet message bearer service in IrLAP protocols was started in a general frame format as for radio data transfer. In addition, from the data source server to the destination client it accomplishes a real-time data transfer with Transport Protocol. The final destination clients which receive the data frame returns the ACK message to initial data source station of an application server. The transfer agent of an access point as one hop gateway to mobile clients can send a TAK (Temporary Acknowledgment) message including disconnected buffering state to data source server or
RAK (Recovery Acknowledgement) message including reconnect recovery state to data source server or neighboring access points.

Link bit rate of Infrared is assumed to 1 to $2 \mathrm{Mbps}$ or $4 \mathrm{Mbps}$ by IEEE 802.11 specification $16 \mathrm{Mbps}$ by AIR specification, 1Gbps by ICSA (Infrared Communication Systems Association) Giga-IR specification [3, 4, 7].

The IR PHY uses near-visible light in the $850 \mathrm{~nm}$ to $950 \mathrm{~nm}$ range for signaling purposes. The IR PHY is not directed. That is, the receiver and transmitter do not have to be aimed at each other and do not need a clear line-of-sight. A pair of conformant IR devices would be able to communicate in a typical environment at a range of up to about $10 \mathrm{~m}$. This standard allows conformant devices to have more sensitive receivers, which may increase range by about $20 \mathrm{~m}$. The IR PHY will operate only in indoor environments. IR radiation does not pass through walls, and is significantly attenuated passing through most exterior windows.

The basic access rate is based on $1 \mathrm{Mb} / \mathrm{s} 16-\mathrm{PPM}$ modulation. The enhanced access rate is based on $2 \mathrm{Mb} / \mathrm{s}$ 4-PPM.

We generally represent an optical wireless network system with a graph model, $\mathrm{G}(N, L)$ with nodes and links (or edges). A set of nodes is described as $\mathrm{N} \underline{\underline{\text { def }}}$ $\left\{n_{0}, n_{1}, \cdots n_{\mathrm{k}-1}\right\}$ for the number of nodes is $\mathrm{k}$ and a set of links as $\mathrm{L} \stackrel{\text { def }}{=}\left\{\mathrm{L}_{0}, \mathrm{~L}_{1}, \cdots \mathrm{L}_{\mathrm{k}-1}\right\}$ where $\mathrm{L}_{\mathrm{i}}$ indicates each set of node $\mathrm{i}$ and $\mathrm{L}_{\mathrm{i}} \stackrel{\text { def }}{=}\left\{l_{0}, l_{1}, \cdots l_{\mathrm{m}-1}\right\}$ for $\mathrm{L}_{\mathrm{i}} \in \exists \mathrm{L}$ and $0 \leq i \leq k$. We can also represent a set of link connections $\mathrm{E}$ as $\mathrm{E}=\sum_{i, j=0}^{m-1} \overline{l_{i} l_{j}}$ for $\mathrm{i} \neq \mathrm{j}$ and $\mathrm{q} \neq r, l_{\mathrm{i}} \in \exists \mathrm{L}_{q}, l_{j}$ $\in \exists \mathrm{L}_{\mathrm{r}}$. A set of time is shown as $\tau=\left\{t_{0}, t_{1}, \cdots, t_{p}\right\}$. Here $t_{0}$ is to start time, $\delta_{0}$ to short threshold time and $\delta_{1}$ to long threshold time. Now we can classify link attributes into three (3) categories as the following Lemmas.

LEMMA 3.1. Let the link state described as the following formal logic description be "Successful connection". The "Successful Connection Link $\left(\mathrm{L}_{\mathrm{S}}\right)$ " means that the connection status of the physical link is good during specific time from $t_{0}$ to $\delta_{0}$.

$$
\square\left(\exists \alpha \in \mathrm{L}_{\mathrm{i}} \wedge \mathrm{L}_{\mathrm{j}} \mid \mathrm{i} \neq \mathrm{j}, \alpha \in \mathrm{E} \text { and } t_{0} \leq t_{0}+\delta_{0}\right)
$$

LEMMA 3.2. Let the link state described as the following formal logic description be "Temporary Disconnection". The "Temporary Disconnection Link (LT)" indicates that the connection status of the physical link is temporarily disconnected in transient time.

$$
\neg\left(\square\left(\exists \alpha \in \mathrm{L}_{\mathrm{i}} \wedge \mathrm{L}_{\mathrm{j}} \mid \mathrm{i} \neq \mathrm{j}, \alpha \in \text { E and } t_{0}+\delta_{0} \leq t_{0}+\delta_{1}\right)\right)
$$

LEMMA 3.3. Let the link state described as the following formal logic description be "Permanent Disconnection". The "Permanent Disconnection Link $\left(\mathrm{L}_{\mathrm{p}}\right)$ " is permanently to break the connection status of physical link. 


$$
\neg\left(\diamond\left(\exists \alpha \in \mathrm{L}_{\mathrm{i}} \wedge \mathrm{L}_{\mathrm{j}} \mid \mathrm{i} \neq \mathrm{j}, \alpha \in \mathrm{E}, t_{0}+\delta_{0} \leq t_{0}+\delta_{1}\right)\right)
$$

This paper splits a total set of link connectivity to three classes as successful connection links, temporary disconnection link and permanent disconnection link shown as Fig. 3. The "Successful Connection Link $\left(\mathrm{L}_{\mathrm{S}}\right)$ " means that the connection status of the physical link is good at a specific time. The "Temporary Disconnection Link $\left(\mathrm{L}_{\mathrm{T}}\right)$ " indicates that the connection status of physical link is temporarily disconnected during the transient time. The "Permanent Disconnection Link $\left(\mathrm{L}_{\mathrm{P}}\right)$ " is permanently to break the connection status of physical link. Here $\delta_{o}$ is to short threshold value and $\delta_{n}$ to long threshold value. This is shown as:

$$
\begin{aligned}
& \mathrm{L}=\left\{\mathrm{L}_{1}, \mathrm{~L}_{2}, \mathrm{~L}_{3} \cdots, \mathrm{L}_{i} \cdots, \mathrm{L}_{\mathrm{n}}\right\}=\sum_{i=1}^{n} \mathrm{~L}_{i} \\
& \mathrm{~L}_{\mathrm{S}}=\left\{\mathrm{L}_{i} \in \mathrm{L}, \text { if } \text { Timer }_{i} \text { of } M C_{i} \leq \delta_{\mathrm{o}}\right\} \\
& \mathrm{L}_{\mathrm{T}}=\left\{\mathrm{L}_{i} \in \mathrm{L}, \text { if } \delta_{\mathrm{o}}<\text { Timer }_{i} \leq \delta_{\mathrm{n}}\right\} \\
& \mathrm{L}_{\mathrm{P}}=\left\{\mathrm{L}_{i} \in \mathrm{L}, \text { if } \delta_{\mathrm{n}}<\text { Timer }_{i}\right\} \\
& \mathrm{L}=\cup\left\{\mathrm{L}_{\mathrm{s}}, \mathrm{L}_{\mathrm{T}}, \mathrm{L}_{\mathrm{p}}\right\}=\left\{\mathrm{L}_{\mathrm{s}} \cup \mathrm{L}_{\mathrm{T}} \cup \mathrm{L}_{\mathrm{p}}\right\}
\end{aligned}
$$

The sender (or primary station) of the existing protocol awaits the answer back (or ACK) from its own addressee (or secondary station; receiver). If it passes the threshold value of schedule time, it regards that it will not be able to accomplish a continuous transmission as the link connection and discontinue connection to the 2nd layer.

The proposed agent technique depends not on just the answer back latency time for judging the presence of data link discontinuation but assumes various link failures based on error and flow control of physical layer or data link layer (or the 2nd layer). The physical layer or data link layer delivers transmission contextual information, whether the permanent discontinuation or a temporary discontinuation, to the upper layer protocol or to the service users differently from the existing message of discontinuation preparation warning.

This error information supports the advancement of data transfer efficiency and service quality by providing the selective transmission backup (including memory buffering) depending on the final selection of user or high position protocol. Additionally, the proposed agent method requires additional mechanisms and concepts as follows.

When temporary disconnection springs up, a relaying sender reports its state or TAK (or temporary acknowledgment) to the initial sender or its upper layers. The overhead of the TAK control message, including disconnection start packet/frame number, can be minimized by a message piggybacking method.

And when the disconnected link is recovering, a relaying sender reports its state or CRI (Consecutive Restore Inform) message to receiver or its upper layers.
If the reconnected communication is completed successfully, a relaying sender informs with an ACK message with its state to initial sender or its upper layers. The overhead of ACK/CRI control message including reconnection start packet/frame number can be optimized using message piggybacking.

How we can manage the refresh cycle of agent data buffer and backup?

FCFS (First Come First Served) method is that firstly older data are deleted if the allocated buffer space or disk space is full. This is a sequential processing technique. As another a-periodic method, we immediately delete the targeted data if it receives an explicit invalid command from sender or receiver.

\section{OPTICAL WIRELESS ACCESS POINT TRANSFER AGENT}

As Fig. 2, this research is the agent method that improves flow and an error control protocol in which is a data link layer IrLAP or an application network layer IrLAN in IrDA/IrSimple transmission protocol stacks.

The data service coverage of an optical wireless network will be able to divide within a short-range area transmission and long-range wide area transmission according to the transmission range size. This research is focusing on the improvement of frame transmission flow and error control based on a short-range area (IrLAN/LAN) packet over a data link layer, IrLAP layer as Fig. 2. The proposed method will be able to be applied to an optical wireless Internet or long-range wide area transmission system from easy extension of a short-range area transmission system.

At the optical wireless network, a mobile client is connecting and then demanding its data in an application server. They are delivered without packet loss or time delay to be transferred to the requested packet frames at real-time through the base station in the condition of optical wireless channel which dynamically changes.

The mobile user dynamically changes location, direction and speed within optical wireless access point as time flows. This paper applies an agent technique based on access point location information AP'position $\left(\mathrm{MC}_{m}\right)$ of radio terminal $\mathrm{MC}_{m}$. Each access point assumes that it is composed of the unit of the optical wireless cell.

As Fig. 4, the proposed AP based agent modules are organized into two flows of initial connected flow and roaming reconnected flow. Each AP agent is operating a pre-buffering function for relaying from data server or its disk storages into out sending buffer and pushing on the connected link for a mobile client $\left(\mathrm{MC}_{i}\right)$ within itself. Then, if the data transfer for a $\mathrm{MC}_{i}$ is halted by link disconnection, its following data flows are abandoned or stored into temporary space in its AP by the 


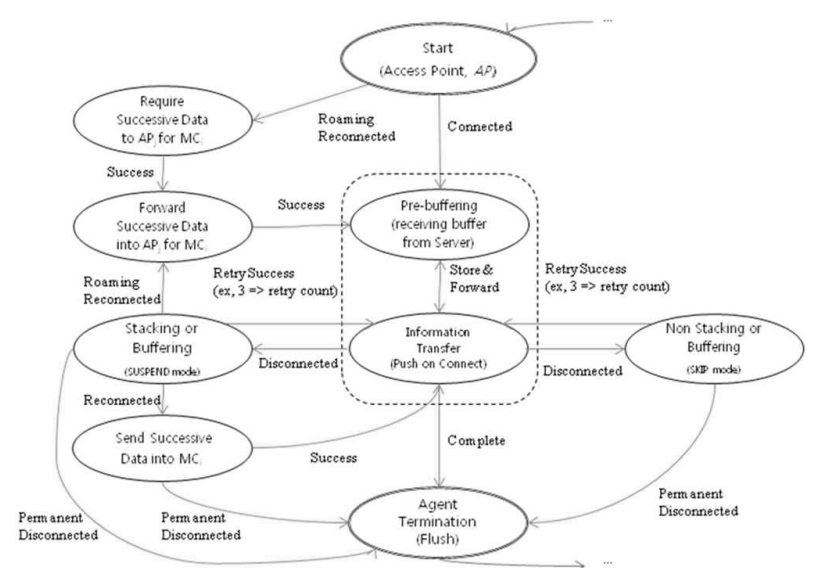

FIG. 4. The proposed access point transfer agent state transition diagram.
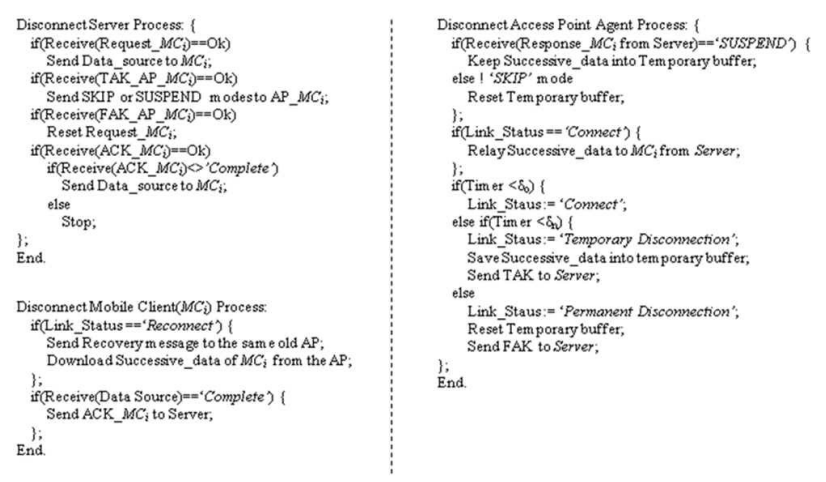

FIG. 5. Disconnect access point agent module.

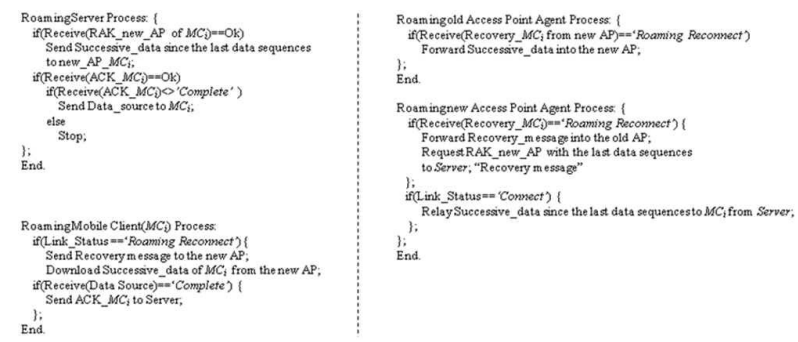

FIG. 6. Roaming access point agent module.

selection of SKIP or SUSPEND mode, respectively, among buffering modes. After disconnection, if the disconnected $\mathrm{MCi}$ reconnects to the same $\mathrm{AP}$, the $\mathrm{AP}$ agent recovers consecutive data of the $\mathrm{MC}_{i}$ from its buffer and if it reconnects to the new AP, the new AP agent recovers consecutive data of the $\mathrm{MC}_{i}$ from buffer of old AP. This agent transfer process can be repeated until data transfer is completed or aborted.

Each access point $\mathrm{AP}_{i}$ has the transmission agent TA (TA: Transfer Agent) to support data transfer caching and buffering. The default transfer agent is initially executed for setup as the real-time transmission agent in each access point. The transmission agent $\mathrm{TA}_{i}$ of each base station supports an agent buffering module and roaming forwarding module dependent on disconnection variable and recovery re-connection location of $\mathrm{MC}$, respectively as follows.

Firstly, network disconnection is a condition for which the mobile client which receives the real-time packet does not appear in any cell before time delay shorter than critical threshold value $\left(\delta_{\mathrm{n}}\right)$ after it disappears without disconnection commands. This research proposes agent buffering techniques to minimize the real-time transmission packet loss by the disconnection of the optical wireless network as Fig. 5.

The agent buffering module selectively supports for transmission efficiency and prevents packet loss which appears via network discontinuation of the radio terminal. In the case that the mobile client which exists in optical wireless access point was discontinued, a transmission agent selectively operates the two buffering techniques of SKIP mode (buffering off mode) with loss permission and SUSPEND mode (buffering on mode) with loss prevention about the transmitting packet. From server to client, the real-time download transmission packet loss of UDP-like transmission modules for transmission efficiency on the OSI $4^{\text {th }}$ layer occurs, which is identical with SKIP modes without buffering. The $4^{\text {th }}$ layer TCP-like transmission with the reliable channel demands the many confirmation messages. It appears the problem that the transmission efficiency comes to be bad suddenly. We support SUSPEND modes with buffering to overcome this problem during a network disconnection.

Secondly, the reconnect operation in an optical wireless cell network is a condition for which a specific mobile client appears in new $A P$ before delaying more than threshold value $\left(\delta_{\mathrm{n}}\right)$ after disappearing from old $A P$. This research provides the predictive pre-fetch technique to minimize the time delay of the real-time packets which occur in the re-connection process as Fig. 6.

The prediction pre-fetch module is a process that brings succeeding packets in advance from the application server because of the fact that the mobile client $\mathrm{MH}$ will reconnect in the near future as it reconnects the changed access point after it disconnects in the middle while it receives packets.

\section{SYSTEM ANALYSIS}

This work assumes that analysis model has three (3) level hierarchy architecture of server (S), access point (AP) and mobile client (MC) for transmission efficiency improvement. The requested data of mobile client are transferred from $\mathrm{S}$ to $\mathrm{AP}$, from $\mathrm{AP}$ to $\mathrm{MC}$.

The transferring process between AP and MC is split into three sub-processes from the AP to MC as follows. First case, after disconnection between $\mathrm{AP}$ and MC, MC 
reconnects at the same old $\mathrm{AP}$ and receives consecutive request data within the threshold time. Second case, after disconnection between the $\mathrm{AP}$ and $\mathrm{MC}, \mathrm{MC}$ reconnects at the other new AP and continuously receives the request data within the given threshold time. The last case is that the disconnection is not recovered permanently and reconnection is not accomplished at any AP.

The following sequences are the experimental variables for system analyzing.

- $\alpha_{0}(\mathrm{bps})$ : Data transfer speed from server $(\mathrm{S})$ to the same old AP

- $\alpha_{n}(\mathrm{bps})$ : Data transfer speed from server (S) to a new AP

- $\beta_{0}(\mathrm{bps})$ : Data transfer speed from the same old AP to MC

- $\beta_{n}(\mathrm{bps})$ : Data transfer speed from new AP to $\mathrm{MC}$

- $\gamma($ bps $)$ : Data transfer speed from old AP to new AP

- $\tau_{\alpha o}(\mathrm{bps})$ : Data propagation delay from server (S) to the same old AP

- $\tau_{\beta o}(\mathrm{sec})$ : Data propagation delay from the same old AP to MC

- $\tau_{\alpha n}(\mathrm{sec})$ : Data propagation delay from server $(\mathrm{S})$ to new AP

- $\tau_{\beta n}(\mathrm{sec})$ : Data propagation delay from new AP to $\mathrm{MC}$

- $\rho_{s}$ : The connection probability that configures successful link between AP and MC

- $\rho_{f}$ : The disconnection probability that configures failure link between AP and MC

- $\rho_{r}$ : The reconnection probability that configures recovery link between the same $\mathrm{AP}$ and $\mathrm{MC}$

- $\rho_{p}$ : The roaming probability that a MC reconnects at a new AP after it disconnects from old AP

- $S$ (byte) : The size of data transferred in all AP

This analysis compares the proposal connectivity model and previous connectivity model in two aspects of disconnection model and roaming reconnection model based on above variables.

In the former disconnection model, data propagation time of the previous $\left(\tau_{\text {pre dis }_{-}}\right)$and the proposal $\left(\tau_{\text {this dis }}\right)$ is as the following equations.

$$
\begin{aligned}
& \tau_{\alpha o} \text { (propagation delay from S to AP) }=\frac{\text { s byte }}{\alpha_{0} \text { bps }}=8 \frac{\mathrm{s}}{\alpha_{0}}(\mathrm{sec}) \\
& \tau_{\beta 0} \text { (propagation delay from AP to MC) }=\frac{\mathrm{s} \text { byte }}{\beta_{\mathrm{o}} \text { bps }}=8 \frac{\mathrm{s}}{\beta_{\mathrm{o}}}(\mathrm{sec}) \\
& \tau_{\text {pre_dis }}=\rho_{\mathrm{s}} \times\left(8 \frac{\mathrm{s}}{\alpha_{0}}+8 \frac{\mathrm{s}}{\beta_{0}}\right)+\rho_{\mathrm{f}} \times\left(2 \times 8 \frac{\mathrm{s}}{\alpha_{0}}+8 \frac{\mathrm{s}}{\beta_{\mathrm{o}}}\right)(\mathrm{sec}) \\
& \tau_{\text {this_dis }}=\rho_{\mathrm{s}} \times\left(8 \frac{\mathrm{s}}{\alpha_{0}}+8 \frac{\mathrm{s}}{\beta_{\mathrm{o}}}\right)+\rho_{\mathrm{f}} \times \rho_{\mathrm{r}} \times 8 \frac{\mathrm{s}}{\beta_{0}}+\rho_{\mathrm{f}} \times\left(1-\rho_{\mathrm{r}}\right) \times\left(2 \times 8 \frac{\mathrm{s}}{\alpha_{0}}+8 \frac{\mathrm{s}}{\beta_{0}}\right)(\mathrm{sec})
\end{aligned}
$$

Then the packet loss ratios indicate the volume of loss data relative to the volume of total data as follows.

$$
\text { The packet loss ratio }=\frac{\text { The volume of loss data }}{\text { The volume of total data }}
$$

In the case that the size of transferred data is S Byte, the packet loss ratios of the previous $\left(\epsilon_{\text {pre }_{-} \text {dis }}\right)$ and the proposal $\left(\epsilon_{\text {this_dis }}\right)$ is as the following equations.

$$
\begin{aligned}
& \varepsilon_{\text {pre_dis }}=\frac{\rho_{\mathrm{f}} \times \mathrm{S}}{\rho_{\mathrm{S}} \times \mathrm{S}+\rho_{\mathrm{f}} \times \mathrm{S}}=\frac{\rho_{\mathrm{f}}}{\rho_{\mathrm{S}}+\rho_{\mathrm{f}}} \\
& \varepsilon_{\text {this_dis }}=\frac{\rho_{\mathrm{f}} \times\left(1-\rho_{\mathrm{r}}\right) \times S}{\rho_{\mathrm{S}} \times S+\rho_{\mathrm{f}} \times \rho_{\mathrm{r}} \times S+\rho_{\mathrm{f}} \times\left(1-\rho_{\mathrm{r}}\right) \times S}=\frac{\rho_{\mathrm{f}} \times\left(1-\rho_{\mathrm{r}}\right)}{\rho_{\mathrm{S}}+\rho_{\mathrm{f}} \times \rho_{\mathrm{r}}+\rho_{\mathrm{f}} \times\left(1-\rho_{\mathrm{r}}\right)}
\end{aligned}
$$

In the latter roaming reconnection model, the data propagation time of the previous $\left(\tau_{\text {pre dis }}\right)$ and the proposal $\left(\tau_{\text {this_dis }}\right)$ is as the following equations. If the reconnected new $\mathrm{AP}$ of $\mathrm{MC}$ requires continuous data to the old AP among neighboring APs, the old AP forwards continuous data to the new AP.

$$
\begin{gathered}
\tau_{\text {an }} \text { (propagation delay from S to new AP) }=\frac{\mathrm{S} \text { byte }}{\alpha_{\mathrm{n}} \mathrm{bps}}=8 \frac{\mathrm{S}}{\alpha_{\mathrm{n}}}(\mathrm{sec}) \\
\tau_{\beta \mathrm{n}} \text { (propagation delay from old AP to new AP) }=\frac{\mathrm{S} \text { byte }}{\gamma \mathrm{bps}}=8 \frac{\mathrm{S}}{\gamma}(\mathrm{sec}) \\
\tau_{\text {pre_re }}=\rho_{\mathrm{s}} \times\left(8 \frac{\mathrm{S}}{\alpha_{\mathrm{o}}}+8 \frac{\mathrm{S}}{\beta_{\mathrm{o}}}\right)+\rho_{\mathrm{f}} \times\left(8 \frac{\mathrm{S}}{\alpha_{\mathrm{o}}}+8 \frac{\mathrm{S}}{\alpha_{\mathrm{n}}}+8 \frac{\mathrm{S}}{\beta_{\mathrm{n}}}\right)(\mathrm{sec}) \\
\tau_{\text {this_re }}=\rho_{\mathrm{s}} \times\left(8 \frac{\mathrm{S}}{\alpha_{\mathrm{o}}}+8 \frac{\mathrm{S}}{\beta_{\mathrm{o}}}\right)+\rho_{\mathrm{f}} \times \rho_{\mathrm{p}} \times\left(8 \frac{\mathrm{S}}{\gamma}+8 \frac{\mathrm{S}}{\beta_{\mathrm{n}}}\right)+ \\
\rho_{\mathrm{f}} \times\left(1-\rho_{\mathrm{p}}\right) \times\left(8 \frac{\mathrm{S}}{\alpha_{\mathrm{n}}}+8 \frac{\mathrm{S}}{\beta_{\mathrm{n}}}\right)(\mathrm{sec})
\end{gathered}
$$

In the case of data size ( $\mathrm{S}$ byte), the packet loss ratios of the previous $\left(\epsilon_{\text {pre }_{-} \text {dis }}\right)$ and the proposal $\left(\epsilon_{\text {this_dis }}\right)$ is as the following equations.

$$
\begin{aligned}
& \varepsilon_{\text {pre_re }}=\frac{\rho_{\mathrm{f}} \times S}{\rho_{\mathrm{s}} \times \mathrm{S}+\rho_{\mathrm{f}} \times \mathrm{S}}=\frac{\rho_{\mathrm{f}}}{\rho_{\mathrm{s}}+\rho_{\mathrm{f}}} \\
& \varepsilon_{\text {this_re }}=\frac{\rho_{\mathrm{f}} \times\left(1-\rho_{\mathrm{p}}\right) \times \mathrm{S}}{\rho_{\mathrm{S}} \times S+\rho_{\mathrm{f}} \times \rho_{\mathrm{p}} \times \mathrm{S}+\rho_{\mathrm{f}} \times\left(1-\rho_{\mathrm{p}}\right) \times \mathrm{S}}=\frac{\rho_{\mathrm{f}} \times\left(1-\rho_{\mathrm{p}}\right)}{\rho_{\mathrm{s}}+\rho_{\mathrm{f}} \times \rho_{\mathrm{p}}+\rho_{\mathrm{f}} \times\left(1-\rho_{\mathrm{p}}\right)}
\end{aligned}
$$

It is assumed that Infrared has bit rates of 1 to 2 Mbps or $4 \mathrm{Mbps}$ according to the IEEE802.11 specifications. The IR PHY uses near-visible light in the 850 $\mathrm{nm}$ to $950 \mathrm{~nm}$ range for signaling. The IR PHY is not directed. That is, the receiver and transmitter do not have to be aimed at each other and do not need a clear line-of-sight. A pair of conformant IR devices would be able to communicate in a typical environment at a range of up to about $10 \mathrm{~m}$. This standard allows conformant devices to have more sensitive receivers, and this may increase range of up to about $20 \mathrm{~m}$. The IR PHY will operate only in indoor environments. IR radiation does not pass through walls, and it is significantly attenuated passing through most exterior windows. 


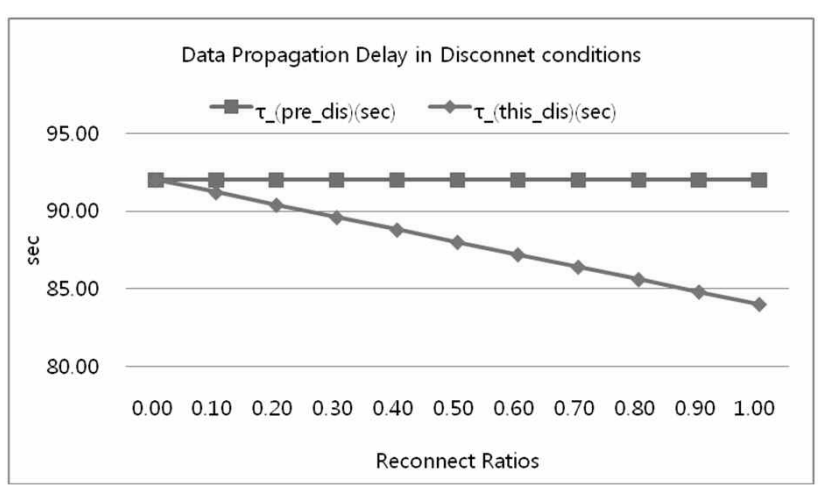

FIG. 7. Data propagation delay in disconnect.

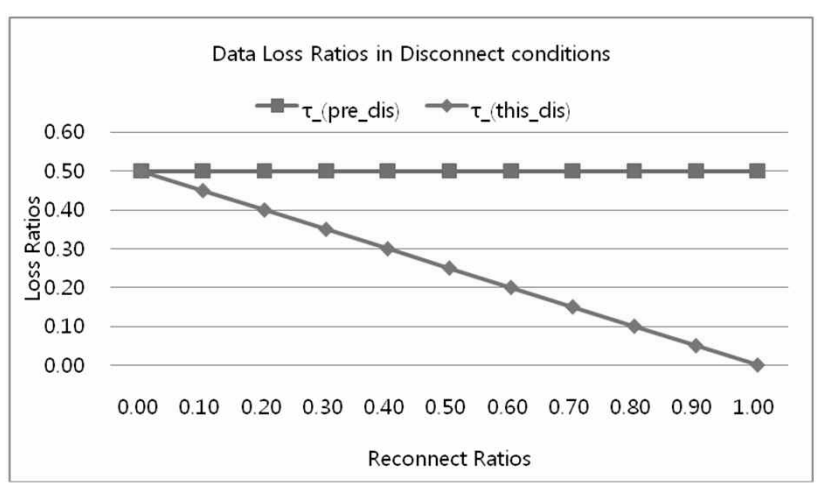

FIG. 8. Data loss ratios in disconnect.

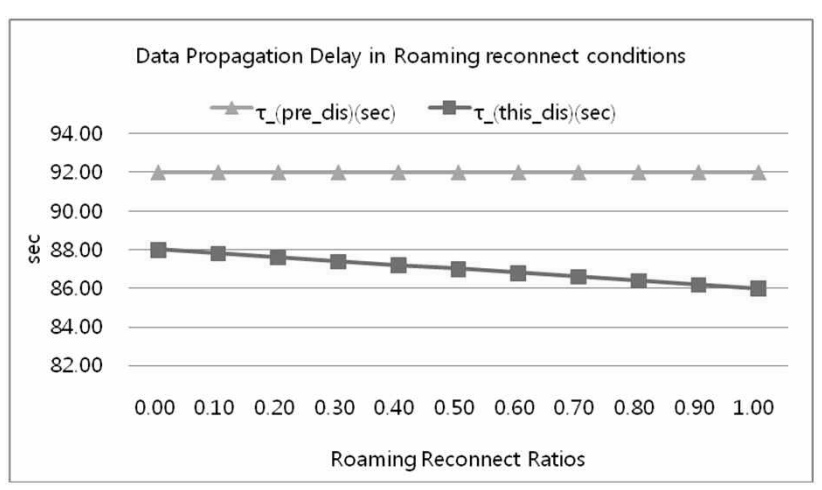

FIG. 9. Data propagation delay in roaming reconnects.

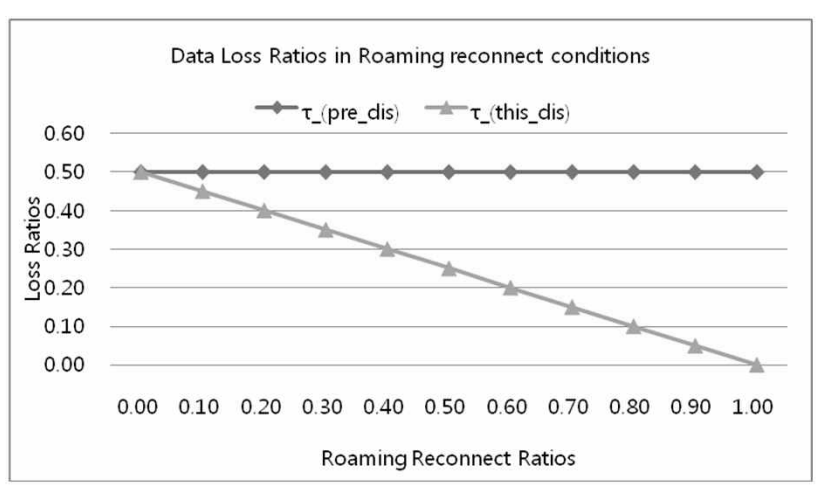

FIG. 10. Data loss ratios in roaming reconnect.
Here we apply the following examples for performance evaluation using equations of (7), (8), (9), (10), (13), (14), (15) and (16) based on the assumptions above. In the first disconnection case, when $\mathrm{S}$ is to $10 \mathrm{Mbyte}$, $a_{o}$ to $10 \mathrm{Mbps}, \beta_{\mathrm{o}}$ to $1 \mathrm{Mbps}$ and $\rho_{\mathrm{s}}$ to 0.5 , the data propagation delay times of sec unit and the data loss ratios are represented in two figures of Fig. 7 and Fig. 8 , respectively, by the variations of reconnect ratio, $\rho_{\mathrm{r}}$.

Figure 7 shows the results of comparing the data propagation delay of the proposal method $\left(\tau_{\text {pre_dis }}\right)$ with reconnect recovery and that of the previous $\left(\tau_{\text {this_dis }}\right)$ without reconnect when a $\mathrm{MC}$ is disconnected in one AP based on equation (7) and (8). The greater the reconnect ratios of $\mathrm{MC}$ increase, the greater the proposed method decreases the data propagation times step by step relative to the previous ones.

Figure 8 shows the results of comparing the data loss ratios of the proposal method $\left(\epsilon_{\text {pre_dis }}\right)$ with reconnect recovery and that of the previous $\left(\epsilon_{\text {this_dis }}\right)$ without reconnect when a MC is disconnected in one AP based on equation (9) and (10). In proportion to the reconnect ratios of MC, the proposed method gradually decreases the data loss ratios relative to the previous ones.

In the second roaming reconnect case, when $\mathrm{S}$ is to 10 Mbyte, $a_{o}$ to $10 \mathrm{Mbps}, \beta_{\mathrm{o}}$ to $1 \mathrm{Mbps}, \mathrm{a}_{\mathrm{n}}$ to $10 \mathrm{Mbps}$, $\beta_{\mathrm{n}}$ to $1 \mathrm{Mbps}, \gamma$ to $20 \mathrm{Mbps}$ and $\rho_{\mathrm{s}}$ to 0.5 , the data propagation delay times of sec unit and the data loss ratios represent two figures of Fig. 9 and Fig. 10, respectively, by the variations of roaming reconnect ratio, $\rho_{\mathrm{p}}$.

Figure 9 shows the results of comparing the data propagation delay of the proposal method $\left(\tau_{\text {pre_re }}\right)$ with roaming reconnect recovery and that of the previous $\left(\tau_{\text {this_re }}\right)$ ones without roaming when a $\mathrm{MC}$ is disconnected in one AP based on equation (13) and (14). Along with growing the roaming reconnect ratios of $\mathrm{MC}$, the data propagation delay of the proposed method is dramatically reduced from that of the previous.

Figure 10 shows the results of comparing the data loss ratios of the proposed method $\left(\epsilon_{\text {per_re }}\right)$ with reconnect recovery and that of the previous $\left(\epsilon_{\text {this_ re }}\right)$ without reconnect when a MC is disconnected in old $\mathrm{AP}$ and reconnected into new AP based on equation (15) and (16). In proportion to the reconnect ratios of MC, the proposed method radically decreases the data loss ratios up to zero loss relative to the previous.

The proposed method raises transmission effectiveness and reduces a waste of transmission resources by improving the reconnect transmission path in the same time when it reduces packet loss and packet time delay. The realtime data transfer agent technique in this paper provides a roaming forwarding module and an agent buffering module which shows the features as follows.

As shown in both Fig. 7 and Fig. 9, the data propagation delays are decreased to $88 \mathrm{sec}$ compared with $92 \mathrm{sec}$ 
when reconnect ratio is 0.5 in disconnection conditions of the same access point and minimized into $87 \mathrm{sec}$ compared to $92 \mathrm{sec}$ when the roaming reconnect ratio is 0.5 in roaming reconnection conditions between the two access points. The agent buffering technique at optical wireless access points improves the packet delays under accomplishing the packet buffering within the buffer size for the network disconnection despite communicating nodes use UDP-like transmission protocols which do not provide for reliable communication channels.

As shown in both Fig. 8 and Fig. 10, the data loss ratios are radically reduced into $25 \%$ compared to $50 \%$ when reconnect ratio is 0.5 in disconnection conditions of the same access point and also minimized into $25 \%$ compared to $50 \%$ when the roaming reconnect ratio is 0.5 in roaming reconnection conditions between two access points. It means that the reconnection ratio of 1 can make the loss ratio to zero (0) if the capacity of the buffer is sufficiently large, for example it is unlimited. The real-time transmission roaming forwarding technique informs the re-connection point location of the mobile client and fetches the succeeding data set from old access point. It minimizes the data re-transfer rate and decreases unnecessary forwarding from application servers, thereby cutting down data frame losses through buffering between the disconnection access point and reconnection access point.

Therefore this shows that this proposed transfer agent technique provides maximum successive transfer services of real-time transmission data for the optical wireless terminals by minimizing both the packet time delays and the packet losses.

\section{CONCLUSION}

As shown in the analysis above, the proposed agent transfer methods produce clear advantages in terms of packet loss ratios and data propagation delays. This work minimizes packet losses up to a zero ratio by an AP based buffering method considering the mobile host position in spite of frequent disconnection and roaming of mobile terminals on optical wireless networks. Concurrently, a forwarding method embedded in the AP transfer agent has enlarged data traffic efficiency. The successive data transfer method minimizes the packet delay into the extent of 4 to 7 percent by network disconnection or user mobility.

The future work includes fault tolerant methods and transfer agent method under the RF wireless transfer system. For general transfer system on wired networks, fault tolerant and agent transfer methods will be researched. Especially, optical wireless networks will realize the sparse spot optical wireless local network as new network configuration and service specification on optical wireless terminals with ultra-speeds (10Gbps or 1Tbps).
This research of fundamental link discontinuation will be expanded to cellular phones which have a high real-time mobility. And wireless packets of wireless sending terminals are transformed to an IP based packet of the third layer through wireless data gateway to transfer to wired server or wireless receiving terminal on remote Internet. The Wireless Control Message Protocol (WC MP) on the 3rd layer is used in environments that do not provide an IP bearer. WCMP is used by WDP nodes and Wireless Data Gateways to report errors encountered in processing a datagram. Error reporting of WCMP is similar to that of ICMP on WDP = UDP like layer of Datagram layer [6]. Further it raises the transmission efficiency of TCP and UDP protocols which are a transport layer over the existing TCP/IP applications.

\section{ACKNOWLEDGMENT}

This research was financially supported by the Ministry of Education, Science Technology (MEST) and Korea Industrial Technology Foundation (KOTEF) through the Human Resource Training Project for Regional Innovation.

\section{REFERENCES}

1. "Comparison between a meshed wireless optical access network and a reference LMDS alternative," (Optical Access 2001), ML45553, 01.01 Rev.1.

2. Y. M. Kwon, D. C. Kim, and J. I. Shim, "An embedded wide-band spiral inductor for $10 \mathrm{~Gb} / \mathrm{s}$ optical transceiver applications," J. Opt. Soc. Korea 12, 49-51 (2008).

3. O. Bouchet and et al., "Seventh framework programme theme 3 information and communication technologies (ICT), state of the art - optical wireless," (www.ict-omega. eu, 2008), ICT-213311 OMEGA, Deliverable D4.1.

4. Infrared data association, "Giga-IR high speed optocommunication," (Infrared data communication Giga-IR SIG, 2007), http://www.irda.org/associations/2494/files/ Giga-IR General.pdf.

5. I. I. Kim, R. Stieger, J. A. Koontz, C. Moursund, M. Barclay, P. Adhikari, J. Schuster, E. Korevaar, R. Ruigrok, and C. DeCusatis, "Wireless optical transmission of fast ethernet, FDDI, ATM, and ESCON protocol data using the terra link communication system," Opt. Eng. 37, 3143-3155 (1998).

6. E. Ayanoglu, K. Eng, and M. J. Karol, "Wireless ATM: limits, challenges and proposal," IEEE Personal Communication 3, 19-34 (1996).

7. S. Williams, "IrDA: past, present and future," IEEE Personal Communications 7, 11-19 (2000).

8. D. Axtman, A. Ogus, and J. Reilly, "Infrared data association LAN access extensions for link management protocol, IRLAN," (Extended Systems Incorporated, HewlettPackard Corporation and Microsoft Corporation, Jul. 1997), IrDA Version 1.0. 
9. K. Y. Kim and S. Kim, "Complete tunneling of light via local barrier modes in a composite barrier with metamaterials," J. Opt. Soc. Korea 12, 314-318 (2008).

10. P. Clark and A. Sengers, "Wireless optical networking challenges and solutions," in Proc. IEEE Military Communications Conference (Monterey, CA, USA, Nov. 2004), pp. 416-422.

11. A. D. Vendictis, F. Vacirca, and A. Baiocchi, "Experimental analysis of TCP and UDP traffic performance over infra-structure 802.11 b WLAN," in Proc. 11th Management Committee Meeting (Ghent, Belgium, 2004), COST 279, Technical Document 279 $\mathrm{TD}(04) 033$.

12. V. Vitsas and A. C. Boucouvalas, "Automatic repeat request scheme for infrared wireless communications,"
Elec. Lett. 38, 244-246 (2002).

13. C. H. Nam, S. C. Liew, and C. P. Fu, "An experimental study of ARQ protocol in 802.11 b wireless LAN," in Proc. Wireless Personal Multimedia Communications (Tropical Jewel of the Pacific, Honolulu, Hawaii, Oct. 2002).

14. B. Emmert and A. Binzenh, "Efficient link failure detection and localization using P2P-overlay network," in Proc. IEEE Proceedings of the First International Conference on Availability, Reliability and Security (Vienna University of Technology, Austria, Apr. 2006), pp. 732 - 739 .

15. Infrared data association, http://www.irda.org/associations/ 2494/files/Publications/IrBurst MRD.doc, (IrBurst Research Group, Sep. 2003). 